Forthcoming in Economy \& Society (2018)

\title{
The deferred promise of radical cure: pharmaceutical conjugations of malaria in the global health era
}

Javier Lezaun

Institute for Science, Innovation and Society

School of Anthropology and Museum Ethnography

University of Oxford

64 Banbury Road; Oxford, OX2 6PN

United Kingdom

E-mail: javier.lezaun@insis.ox.ac.uk

\begin{abstract}
This article explores the changing political economy of malaria drug discovery by tracing the career over the last four decades of a single molecule, tafenoquine. First identified as a promising antimalarial by the U.S. Army in the 1970s, tafenoquine has recently been approved by the Food and Drug Administration for the radical cure of vivax malaria - the first product to receive marketing authorization for this indication in more than sixty-five years. The new drug is the result of a collaboration between the pharmaceutical company GlaxoSmithKline and the non-profit organisation Medicines for Malaria Venture, with the financial support of the Bill and Melinda Gates Foundation. The successful development of tafenoquine, the article argues, signals an important transition within the global health era: from the chemotherapeutic humanitarianism that characterized efforts against malaria in the early 2000s, towards a period of drug discovery increasingly driven by a promissory commitment to global disease eradication. The article uses the example of tafenoquine to advance a more general argument about the multiple and evolving pharmaceutical conjugations of malaria - the articulation of competing visions of the disease around the capabilities (and limitations) of particular molecules.
\end{abstract}

Keywords: malaria; global health; drug development; tafenoquine; Krintafel 


\section{The deferred promise of radical cure: pharmaceutical conjugations of malaria in the global health era}

Javier Lezaun

University of Oxford

Pharmaceutical products have always been central to the making and re-making of malaria as a "tropical disease," each new drug advancing the boundaries of therapeutic possibility and redefining the parameters of medical obligation and neglect (Packard 2007). With the end in the 1970s of large-scale international efforts to eliminate malaria by controlling the mosquito vector, and against the backdrop of the still inconclusive search for a highly protective vaccine, antimalarial drugs have served as the first and last line of defense against the disease, the commodity whose chemical characteristics and geographical circulation defines the therapeutic landscape (Baird 2017).

Yet antimalarial drugs have defined this landscape as much by their absence as by their presence. It has been the lack of medicines against specific forms or stages of malaria, the loss of efficacy of mainline drugs due to growing parasite resistance, and the practical inaccessibility of existing medicines to those in need of treatment that have historically shaped clinical outputs for most people around the world. Pharmaceuticals are thus at the heart of malaria's cruel paradox: a treatable disease that continues to kill and incapacitate millions.

This state of therapeutic unavailability is rooted in the peculiar political economy of pharmaceutical research on malaria. For most of the twentieth century, the discovery and development of new antimalarial drugs was driven by the interests and capabilities of two types of actors: governments concerned with the protection of armed forces deployed to malaria-endemic regions, and pharmaceutical companies seeking to market prophylactic products to international visitors to those same regions (Slater 2009). This particular nexus of state and corporate interests made malaria drug discovery a branch of what became known as 'travel medicine,' a regime of research and care that 
prioritized the needs of non-immune visitors from regions of the world where malaria was not a routine concern.

This era of malaria drug discovery entered into a rapid decline in the 1980s. Severe cuts to the research budgets of defense organisations, particularly that of the U.S. Army, limited the ability of Western militaries to support drug development efforts, while many pharmaceutical companies dismantled their malaria R\&D programs to concentrate on more profitable therapeutic areas. By the early 1990s, only a handful of companies maintained active portfolios in malaria, and the aggregate output of their endeavors was paltry. ${ }^{i}$ As the twentieth century came to an end, then, the chemotherapeutic arsenal remained limited and the pharmaceutical pipeline virtually empty, at a time when drug resistance was on the rise in Africa and Asia and the disease appeared out of control in most endemic regions.

The rise at the turn of the century of a new 'global health' agenda focused on the development of new therapeutics was a reaction to what many considered an imminent 'malaria disaster' (White et al 1999). In the new regime, large philanthropic organisations replaced militaries and pharmaceutical companies as the sponsors of last resort for malaria drug discovery. They channeled substantial resources into academic and corporate research, often through the intermediation of "product development partnerships" that aggressively blurred the boundary between public and private domains (McGoey et al 2011; see also Samsky 2012; Ecks and Harper 2013). As these philanthropies, in particular the Bill and Melinda Gates Foundation, became the dominant funders of pharmaceutical research, they also came to exert an outsize influence over the definition of malaria-related medical needs and the choice of the most adequate means of addressing them.

This article examines shifts and continuities in malaria drug discovery since the 1980s by tracing the career over that period of a single molecule, tafenoquine. A member of the 8-aminoquinoline class of antimalarials, tafenoquine has recently been approved by the U.S. Food and Drug Administration (FDA), and is widely expected to play a prominent role in the control and elimination of malaria over the coming decades. The molecule's most distinguishing feature is a rare ability to kill hypnozoites, the form of the 
Plasmodium vivax parasite that lies dormant in the liver and can lead to relapse weeks, months or even years after an infectious mosquito bite. This efficacy against the elusive hypnozoite means that tafenoquine can provide what is known as radical cure of vivax malaria: the complete elimination of parasites from the human body, including those lodged in the liver of an otherwise asymptomatic individual. For this reason, the drug has significant value not only for the clinical treatment of patients, but also for the elimination of the hidden "human reservoir" of latent parasites responsible for recurrent clinical attacks and ongoing transmission.

What makes tafenoquine a particularly useful marker of the historical evolution of malarial drug discovery is how long it has taken the molecule to fulfill its chemotherapeutic promise. Tafenoquine has been in development for forty years, an unusually lengthy period of time even by the protracted timelines of pharmaceutical R\&D. The molecule was first synthesized and tested by the U.S. Army in the 1970s as a potential new treatment option for American forces operating in vivax-endemic areas, but its prospects dissipated with the decline of the Army's antimalarial research program. It was subsequently developed as a new prophylactic against Plasmodium falciparum by the company SmithKline Beecham in partnership with the U.S. Department of Defense (DoD), a collaboration that exemplified the military-pharmaceutical complex in the late period of the travel medicine era. This second phase in the history of the molecule came to an abrupt end at the turn of the century, precisely as the new global health vision was taking hold. Addressing the urgent clinical needs of those living in malaria-endemic regions, not improving prophylactic options for Western travellers, became the paramount concern, and tafenoquine appeared to exemplify the therapeutic and ethical failures of a past era of drug discovery. After a few years stuck in the limbo of abandoned investigational drugs, however, tafenoquine made a triumphant return in 2007, this time repurposed as a radical curative treatment for vivax malaria. In this capacity it quickly became a cornerstone of the new global malaria elimination agenda spearheaded by the Gates Foundation.

The forty years during which tafenoquine has been in development thus encompass the era of travel medicine, the rise of global health as a vital 
humanitarian project, and a current period of malaria pharmacology focused on the population-wide elimination of the parasite. While malaria has been consistently pharmaceuticalized during this period - it has been seen primarily as a human pathology to be tackled by administration of the right antimalarial drug -pharmaceutical strategies have articulated different and often contrasting visions of malaria. In this sense, the development of tafenoquine represents what we could call, after Hecht (2002), a new pharmaceutical conjugation of malaria, the organisation of a particular understanding of the disease around the capabilities (and limitations) of a novel molecule. Through this process of pharmaceutical conjugation, the plans and promises of the actors that at any given time lead the fight against the disease acquire a concrete, chemical form, and it is in that form that those plans and promises succeed or fail. The approval and imminent commercialization of tafenoquine thus materializes a political economy of drug discovery dominated by the priorities of large philanthropic actors and oriented towards the global eradication of the disease. At the same time, it alerts us to the deep, molecule-level continuities that exist between the contemporary configuration of the global health regime and past efforts to take population-wide pharmaceutical measures against the disease.

The analysis that follows is based on over thirty interviews, conducted between 2014 and 2018, with corporate executives, officers in philanthropic organisations and scientific researchers who at one point or another were involved in the development of tafenoquine. Their views have been complemented and in some cases qualified with the help of archival materials and technical publications, in order to allow a fuller reconstruction of the long history of the molecule and of its significance in the evolution of pharmaceutical strategies against malaria.

\section{WR238605 and the decline of military pharmacology}

The chemical compound that would become tafenoquine was first synthesized in 1978 at the U.S. Army's Walter Reed Army Institute of Research (WRAIR), in Silver Spring, Maryland. Known at the time as WR238605, the molecule was one 
of several promising leads that emerged in the 1960s and 1970s from the Army's drug discovery program. This program was a response to the acute challenge that malaria had posed to military operations in Vietnam. War in Southeast Asia had quickly exposed the limitations of the military's two 'wonder drugs,' chloroquine and primaquine. Chloroquine's efficacy was dented by the proliferation of resistant strains of $P$. falciparum. As for primaquine, a drug first deployed by the U.S. Army during the Korean War, its value against P. vivax infection was hampered by a low therapeutic index. Like other 8aminoquinolines, primaquine could cause hemolysis - the destruction of red blood cells - and potentially fatal anemia in individuals with an X-linked genetic predisposition to expressing low levels of the glucose-6-phosphate dehydrogenase (G6PD) enzyme.ii Furthermore, to achieve its full therapeutic effect primaquine required a lengthy administration regimen - typically daily doses over a two-week period - which severely limited compliance with the standard course of treatment.

The shortcomings of primaquine were a particularly sensitive matter for the U.S. military. Primaquine was at the time - and it would remain for more than sixty years - the only drug capable of preventing relapses of vivax malaria by eliminating the latent hepatic form of the parasite, the hypnozoite. As a result, the drug's limitations not only complicated the treatment of infected individuals, but also increased the chances that the hundreds of thousands of military personnel rotating home from Southeast Asia would arrive in the United States with parasites in their liver and import malaria back into the country.

The discovery of a drug that would be 'better than primaquine' thus became a priority for the Army's drug discovery program. WR238605 was a synthetic derivative of primaquine, and it emerged as the most promising candidate from the screening of more than 300,000 compounds. Initial animal studies showed that the molecule had greater potency and less toxicity than primaquine. WR238605 was able to provide causal prophylaxis by preventing parasites from entering the blood stream, and displayed higher activity against hypnozoites than primaquine. Unlike primaquine, moreoever, WR238605 also appeared to be effective against the asexual blood-stage form of vivax and falciparum parasites. Furthermore, it had a strong sporontocidal effect, 
interfering with the development of the parasite within the mosquito when ingested in a blood meal (Coleman 1990).

The most striking pharmacokinetic feature of tafenoquine was its slow metabolism and extended absorption phase. This resulted in a much longer halflife - approximately 14 days against the 4-6 hours of primaquine. The molecule's long-lasting action, combined with its relative safety, raised the possibility of achieving radical cure of vivax malaria with a single dose of the drug, something that would represent a vast improvement over the standard 14-day primaquine regimen (Peters et al 1993).

As pre-clinical studies began to reveal the potential of tafenoquine, however, the Army was quickly losing its capacity to develop the molecule. WRAIR's budget had already begun to shrink towards the end of the Vietnam War, and the withdrawal of U.S. forces from Southeast Asia led to further cuts in the DoD's financial allocation to malaria research. The budgetary situation continued to deteriorate throughout the 1980s. Declining recognition of the strategic threat that malaria posed to U.S. national security, political pressure from Congress to concentrate 'basic' malaria research within the National Institutes of Health, a change of funding priorities in response to the HIV epidemic, and the generalized reduction of defense budgets that followed the end of the Cold War further eroded the sustainability of WRAIR's world-leading antimalarial drug discovery program. By 1991, as tafenoquine was scheduled to enter its first phase I trial, the annual budget for malaria R\&D at WRAIR hovered around US\$3 million, an amount that was clearly insufficient to sustain the clinical evaluation of the Army's most promising compound (U.S. Army 1992).

WRAIR's financial predicament led to a radical change in how the U.S. military participated in malaria drug discovery efforts. Since World War II, the Army had been able to lead the development of its own molecules, commissioning pieces of research from external academic or commercial partners but maintaining overall control of the process all the way to licensure. By the mid-1980s, however, the Army had become wholly reliant on the private sector to conduct clinical trials of its candidate drugs. WRAIR continued to carry out extensive pre-clinical research, even phase I studies with human volunteers (typically servicemen), but would then have to transfer the molecule to an 
industrial partner to complete its clinical evaluation and file an application with the Food and Drug Administration (FDA). The first such agreement was struck with Hoffman-La Roche for the late-stage development of mefloquine (commercialized under the trade name Lariam). Soon afterwards, WRAIR and Smith Kline \& French Laboratories agreed to collaborate on the evaluation of halofantrine (Halfan). After Smith Kline \& French Laboratories merged with the Beecham Group to form SmithKline Beecham in 1989, the new company and WRAIR signed an agreement to develop WR 238605 (now renamed SB-252263, and known also as etaquine).

Although they were sometimes characterized as "co-development partnerships," the agreements that the U.S. Army reached with pharmaceutical companies in the 1980s were markedly different from the public-private malaria drug discovery alliances that would emerge in the late 1990s. In contrast to the intimate and intricate collaborations of the global health era, the agreements between the U.S. military and its industrial partners were essentially one-time business transactions, whereby the Army - and by extension the U.S. Government - handed over control over the relevant molecule to a pharmaceutical company in return from a commitment to register the new drug with the FDA so that it would be available for use by American forces and government personnel. ${ }^{\text {iii }} \mathrm{A}$ former executive at SmithKline Beecham who negotiated the terms of the collaboration with the U.S. Army for the development of tafenoquine describes the contract in question as "very loose": "It essentially said 'We [US Army] have developed this to the point at which we cannot develop it any further. We need an industrial partner. You develop it and you make it available to us.' In other words, the requirement was pure and simple: this must be registered in the US so that it can be used by the troops... That was the only requirement: to register it in the US. It wasn't to be first registered in the US, it just had to be done."

While industry executives describe these agreements as mutually beneficial - as another manager at SmithKline Beecham puts it: "they had the molecules, we wanted to use them; they were only too pleased" - former WRAIR staff members recall the transactions with mixed feelings. In their view, the contracts failed to protect the public interest, specifically as it pertained to the 
provision of price guarantees for the procurement by the government of any drugs that might result from these collaborations. Crucial intellectual property rights held by the U.S. government, as well as pre-clinical and clinical data dossiers, were given away as a bargaining chip to attract the interest of the industrial partner (cf. Croft 2007; Lezaun and Montgomery 2015). A former Director of WRAIR's Division of Experimental Therapeutics laments the failure to use the extensive intellectual property portfolio accumulated by the U.S. Government to extract better terms from pharmaceutical companies. "The things that we take for granted here and now about global access and pricing and things, we didn't do any of that. We were completely naïve because we were desperate."

If a rapidly shrinking budget was the ultimate reason for this desperation, the "loose" and lopsided nature of the agreements also speaks to a lack of business acumen on the part of those responsible for the Army's malaria $R \& D$ program. Despite its limited financial resources, the Army still retained unique drug discovery capabilities. One such resource was its highly calibrated method for testing experimental antimalarial compounds in primates - key pre-clinical studies of tafenoquine's efficacy against liver-stage parasites were conducted on the Army's large colonies of rhesus monkeys, held at the Armed Forces Research Institute of Medical Sciences (AFRIMS) in Bangkok, Thailand, and the Central Drug Research Institute (CDRI) in Lucknow, India. A second unique capability of the Army was its access to large cohorts of research subjects for the evaluation of compounds. This included U.S. military personnel, but also members of allied foreign forces. Clinical studies of tafenoquine would be conducted on Thai soldiers operating along the country's northeastern border (1996-1997), and on Australian forces deployed on UN-sponsored missions to Papua New Guinea (1998-1999) and East Timor (2000-2001).

These extensive testing infrastructures, established in the context of close military-to-military cooperation during the Cold War, were essential to the development of tafenoquine and would have been difficult and costly for any of the Army's corporate partners to replicate. The inability of the Army to translate these resources into more advantageous contracts was also the result of a lack of expertise and legal savvy when it came to negotiating the transfer of technology 
and intellectual property rights. In the words of the former WRAIR Director quoted earlier: "We were very mission driven, very altruistic, really babes in the wood when it came to business and such, and it showed, right? We routinely got taken advantage of and didn't understand the operating environment."

\section{The military-industrial complex in the era of travel medicine}

On 19 December 1991, SmithKline Beecham submitted an Investigational New Drug application to the FDA for the clinical evaluation of WR238605. The proposed program of clinical investigation reflected the transition of the molecule from its military origins to a new commercial context. SmithKline Beecham was attracted by the prospect of developing WR238605 as a prophylactic against falciparum malaria for short-term visitors to malariaendemic regions. Pre-clinical studies appeared to bear out this prospect: WR238605 was able to prevent the emergence of blood-stage P. falciparum parasites, and its long-lasting action suggested that a single 'loading' dose of the molecule administered prior to exposure might be sufficient to maintain this protection for 2 to 3 weeks, the average duration of business or tourist travel to endemic areas. The development of such a prophylactic represented a considerable commercial opportunity - studies used by the industry at the time estimated a market of 25-30 million annual short-term visitors from Western countries to malaria-endemic regions. P. falciparum was the most virulent species of the parasite, and the dominant one in sub-Saharan Africa, where SmithKline Beecham had extensive marketing networks.

While the U.S. Army shared an interest in the prophylactic indication of the molecule, it remained committed to its development as a radical treatment for vivax malaria. This had been the crucial factor in the selection of tafenoquine as the candidate to replace primaquine, and the threat of relapsing infections among troops operating in vivax-endemic regions continued to be at the forefront of the military's concerns. In the aftermath of Operation Restore Hope, the deployment of U.S. troops to Somalia in 1992, more than two hundred service members experienced relapses of $P$. vivax infection after their return - 
the largest outbreak of imported malaria in the United States since the Vietnam War. The outbreak revealed not only the continuing difficulty of ensuring adherence to the standard primaquine regimen, even among military personnel, but also the extent of parasite resistance to the drug - a significant proportion (43 per cent) of the soldiers who received the 14-day course of primaquine after their return to the U.S. went on to experience relapse (Smoak et al 1997).

The experience in Somalia, and the growing emphasis during this period on long-term peacekeeping missions, altered the U.S. Army's thinking on malaria control. In addition to treating infected personnel, the Army increasingly considered the possibility of "shutting down malaria epidemics where military deployments might occur in areas in [sic] intense transmission" (Milhous 2001). In the case of vivax-endemic areas, eliminating malaria during military operations would require administering drugs to local residents to wipe out the reservoir of liver-stage parasites.

Tafenoquine was the only candidate then in development able to perform this duty. Yet the clinical evaluation programme designed by SmithKline Beecham focused on the efficacy of the molecule as a prophylactic against $P$. falciparum parasites, and it was this indication that was investigated in the studies sponsored by the company. A first randomized, double-blind, placebocontrolled phase II trial with healthy adults was carried out in western Kenya in 1997. A second study followed in northern Ghana in 1998. A third dose-raging study with young adults (12-20 years of age) took place in Lambaréné, Gabon, in 1999. A fourth study started in 2000 in the Nyanza Province of western Kenya. All these trials evaluated quick regimens, typically three consecutive daily doses of tafenoquine followed by weekly administration of the drug for the maintenance of prophylactic effect. The studies, in other words, evaluated modalities of administration that would suit Western travelers visiting hightransmission areas for relatively briefs period of time.

The expectation that Western travelers would be the main users of the drug, and that the drug would be prescribed prior to arrival in an endemic area, allowed these studies to circumvent, at least temporarily, the molecule's most critical safety challenge: its toxicity in individuals with low levels of the G6PD enzyme. It was assumed that international travelers would have access to 
professional laboratory-based diagnosis of their G6PD status in their home countries, and that they would be screened prior to the administration of the drug. The trials conducted in Africa in the 1990s only included individuals with normal levels of G6PD activity, and the screening of participants followed standard laboratory protocols. As a result, key pharmacological questions, such as the relationship between dose and toxic effect in different variants of the G6PD deficiency genotype, the hemolytic risk associated with 'moderate' or 'intermediate' levels of enzymatic activity (i.e. women heterozygous for a G6PD deficiency allele and a G6PD normal allele), or the manner in which the G6PD status of individuals could be diagnosed under field conditions, remained unaddressed.

A further exclusion criterion affected pregnant women - even those considered 'G6PD normal' - since the G6PD status of the fetus could not be known. Each trial addressed this issue differently. The first study in Kenya excluded women who "were pregnant or unwilling to avoid pregnancy"'” (Shanks et al 2001). In the trial conducted in northern Ghana, only women between the ages of 50 and 60 were enrolled, and after they had tested negative for pregnancy in a urine test (Hale et al). The study of young adults conducted in Gabon excluded pregnant or lactating women; all female participants were tested for pregnancy, and in some cases were offered free condoms (Lell et al 2000). The second Kenyan study excluded “women who were pregnant or lactating or who in the opinion of the investigator were at risk of becoming pregnant."iv

In sum, of the several possible uses of tafenoquine, then, one emerged by the end of the decade as "the most obvious one - protecting the short-term traveler going to an area where malaria is endemic" (Shanks et al 2001: 1973). The trials conducted in West and East Africa in the 1990s had all assessed this indication. Excluded from the program of clinical evaluation were pregnant women (a category sometimes defined expansively to encompass a large proportion of women of childbearing age) and young children, the two groups most vulnerable to severe falciparum malaria. It was at this moment, however, when tafenoquine appeared poised to become "a major new antimalarial for the new millennium" (Peters 1999: 351), that changes in the corporate landscape, 
Forthcoming in Economy \& Society (2018)

and a radical shift in the international politics of malaria control, brought its development to an unexpected halt.

\section{The rise of chemotherapeutic humanitarianism}

The late 1990s witnessed a wave of consolidation in the pharmaceutical industry, leading to what some observers described as a new "global oligopoly" in the sector (Galambos 2001). In 2000, SmithKline Beecham merged with Glaxo Wellcome to create GlaxoSmithKline (GSK). Before the merger, Glaxo Wellcome had registered its fixed-dose combination of atovaquone and proguanil for the treatment and prophylaxis of falciparum malaria. This drug, which would be commerdialised under the trade name Malarone, would give GSK a firm hold over the market for malaria prophylactics in many Western countries. Malarone was, moreover, an expensive drug, costly to manufacture and priced out of the reach of most people in malaria-endemic countries. With an effective - and profitable - falciparum prophylactic in its portfolio, the stakes in the development of tafenoquine quickly diminished within the newly merged company. ${ }^{v}$

The internal reorganization that followed the merger also complicated tafenoquine's prospects. At SmithKline Beecham the molecule had been the responsibility of the International Commercial Division, which operated semiindependently of the central R\&D arm of the company. At GSK it fell under the remit of an integrated R\&D Division. Its new managers decided to take a second look at the molecule's safety data, concerned by newly obtained evidence of renal toxicity in rats, and by reports of adverse ophthalmological effects among Australian soldiers enrolled in a phase III trial in East Timor. ${ }^{\mathrm{vi}}$ To allay these concerns GSK and the U.S. Army Medical Research and Materiel Command launched in 2001 two new safety trials. Although these studies, conducted in the UK and the US, produced no evidence of clinically relevant toxicity in healthy individuals exposed to weekly $200 \mathrm{mg}$ doses of the molecule over a six-month period, they effectively returned the molecule to phase I status, further delaying its development. As a former tafenoquine project leader at SmithKline Beecham 
ruefully notes, these trials "put another stop for about three years, while they sort of sat and watched patients not go blind."

While tafenoquine languished in protracted clinical studies and reevaluations of its commercial merit within the newly created GSK, the international landscape of malaria control was changing beyond recognition. In 1998 the WHO, the World Bank, UNICEF and UNDP had launched the Roll Back Malaria initiative with the goal of halving malaria deaths by 2010. In 1999 the product development partnership Medicines for Malaria Venture (MMV) was created with the mission of spearheading the discovery of new medicines to address unmet clinical needs, and the support of several philanthropic organisations and international development aid agencies. That same year, the William H. Gates Foundation - the forerunner of the Bill and Melinda Gates Foundation - gave its first large grant to the Malaria Vaccine Initiative. A year later the Foundation entered the field of malaria drug discovery with a $\$ 25$ million award to MMV, immediately becaming the partnership's largest financial sponsor.

These new actors not only injected significant resources and a sense of urgency into drug discovery efforts, but also introduced a new understanding of what sort of threat malaria represented, what kinds of antimalarial drugs were needed, and who ought to benefit most directly from their development. In their view, the essential medical needs to be addressed were those of the billions of people living in endemic regions of the world, particularly populations in areas of sub-Saharan Africa with high transmission of falciparum malaria. Pregnant women and young children were the most vulnerable groups. The former were at risk of maternal anemia and other complications that often led to low birth weight and increased neonatal mortality. The latter, due to their lack of immunity, were the were most likely to suffer severe and fatal malaria. Of the more than 1 million annual deaths the WHO attributed to malaria in 1999, around 90 per cent (961,000 cases) occurred in Africa, and of those a vast majority (745,000 cases) involved children under four years of age (WHO 1999). The most pressing task was to improve this population's access to existing medicines. As for new drugs, the priorities were more effective ways of treating severe malaria, affordable artemisinin-combination therapies (including new 
pediatric formulations), and better options for intermittent preventive treatment for pregnant women and infants. Improved prophylaxis for visitors to malariaendemic regions was at best a secondary goal, at worst an unjustifiable diversion of much needed financial and scientific resources.

In the words of a former Director of WRAIR's Division of Experimental Therapeutics, prophylaxis quickly became "a dirty word" in key policy circles. "You could not use [the word] prophylaxis in mixed company in the global health environment, because the funders, all they saw is: 'That's spending money for rich Americans to go on holiday in Kenya, and for the military. That's just another tool to enable the imperialistic ambitions of the U.S. military'. It was a very charged debate. MMV and all those groups... you couldn't even talk to them about prophylaxis!"vii

This climate of opinion had a direct correlate in the rapidly changing views on the ethics of clinical research on new malaria therapeutics. The controversies that surrounded HIV drug trials in the 1990s, concerning the use of placebo-treated control groups and the inaccessibility (i.e. unaffordability) of new medicines to the populations on which they had been tested, soon extended to malaria research. Not only did most of the trials for new prophylactics include a placebo group, but they also assessed drug regimens that were of secondary value to those living in malaria-endemic regions. Moreover, they often led to products that were unavailable to trial participants. Malarone was the obvious example: it had been extensively evaluated in countries where the vast majority of the population could not afford the medicine at the price at which it was commercialized.

As a result of these concerns, in 2000 the World Medical Association amended the Declaration of Helsinki, a set of principles governing research involving human subjects, to introduce stricter criteria for the acceptability of clinical trials. In addition to condemning the use of placebos when effective standards of treatment were available, the new Declaration stated that clinical research was only ethically permissible "if there is a reasonable likelihood that the population in which the research is carried out stands to benefit from the results of the research." Even more specifically, "at the conclusion of the study, every patient entered into the study should be assured of access to the best 
proven prophylactic, diagnostic and therapeutic methods identified by the study." In the view of several of the researchers involved in the development of tafenoquine at the time, these amendments gave GSK an additional reason not to pursue the development of the molecule, and provided pharmaceutical companies with a further justification - if a further justification was needed - to abandon work on malaria prophylactics altogether. "Major pharmaceutical companies," wrote three WRAIR scientists, "have interpreted [the amendments] to mean that the traditional development paradigm may be considered unethical because of doubt over the likelihood of benefit to endemic populations participating in clinical studies, the use of placebo, and the sustainability of posttrial access to study medications" (Dow et al 2008).

In addition to increased funding for research and development, then, the new global health era was characterized by a different set of therapeutic priorities and new ethical thresholds for clinical research. The commercial calculations that had sustained malaria drug discovery in the past were dismantled and replaced by a humanitarian obligation to assist those in immediate medical need. The now dominant philanthropic actors were eager to enroll big pharmaceutical companies in this endeavor - former pharma executives often led the public-private partnerships that emerged at the turn of the century - but on the condition that corporate strategies were infused with this new humanitarian ethos. In place of the direct financial interest that had driven antimalarial drug discovery in the previous era, the new global health regime expected companies to embrace - whether out of altruistic motives, for selfish reputational reasons, or for both - a model of corporate citizenship in which research on neglected tropical diseases was presented as an expression of social responsibility (cf. Ecks 2008). Corporate R\&D activities could be directly supported and subsidised by philanthropic donors or development aid budgets, but only after these activities had been separated from the for-profit commercial core of pharmaceutical companies.

Tafenoquine appeared wholly out of step with these trends. Not only was it seen as an old molecule bedeviled by lingering toxicity issues, but it epitomized a model of pharmaceutical research driven by the search for profit and oriented towards the medical needs of affluent customers in the West. Excluded from the 
ethico-therapeutic vision that was inspiring a "renaissance in malaria research" (Winzeler 2008), tafenoquine failed to overcome the final hurdle. The commercial logic that had explained its development as a P. falciparum prophylactic had run its course, while its potential value as an asset in the pharmaceutical arsenal of Western militaries was no longer sufficient to marshal the resources require to complete its development. A former member of WRAIR who had been extensively involved in the evaluation of the molecule in the 1990s describes the situation in the early 2000s as one of impasse, with none of its sponsors sufficiently committed to pushing the molecule past the finish line. "We ended up with a hypersensitive drug company that was basically developing it as a matter of corporate social responsibility - they had no expectations that they were going to make profits - and the military, which was getting it ready for a war that hadn't occurred, and probably wouldn't." Caught between an old era of malaria drug discovery that was coming to an end and a new one that was just getting started, work on tafenoquine slowed down to a halt until, in 2004, GSK decided to suspend the clinical program and shelve the molecule.

\section{The Gates Foundation and the new eradication agenda}

In a landmark speech at the 2007 Gates Malaria Forum, Melinda Gates called for a step change in the global fight against malaria. Reflecting on the progress made in the containment of the disease since the turn of the century, Gates emphasized "the historic opportunity not just to treat malaria or to control it - but to chart a long-term course to eradicate it." She acknowledged that "the word 'eradication' is troubling to many people with deep knowledge of malaria." Yet, she argued, “to aspire to anything less is just far too timid a goal for the age we're in. It's a waste of the world's talent and intelligence, and it's wrong and unfair to the people who are suffering from this disease" (Gates 2007).

The call to re-orient the work of the international community towards eradication caught many by surprise. Some Forum attendees "thought the philanthropist had misspoken" (Roberts and Enserink 2007) when she used the word 'eradication,' that she could not possibly have meant the world-wide 
elimination of the parasite, the technical meaning of the term. Since the failed global eradication campaign of the 1950s and 1960s, the fight against malaria had been limited to the management of clinical cases and the containment of the mosquito vector. By the end of the century, even those goals seemed unduly optimistic. Mortality and morbidity were on the rise, with spotty access to antimalarials in most countries and rising numbers of drug-resistant infections. The two key international initiatives launched at the time, the WHO-sponsored Roll Back Malaria Partnership (1998) and the Global Fund to Fight AIDS, Tuberculosis and Malaria (2002), focused on the distribution of drugs and insecticide-treated bed nets. One can find virtually no reference to malaria elimination, let alone eradication, in the key policy documents of this period.

By the time Melinda Gates delivered her speech, however, enough progress had been made in the control of malaria over the previous years to justify more ambitious goals. The number of deaths attributed to the disease had declined significantly, mostly as a result of the wider use of long-lasting insecticidal nets. On the therapeutic front, artemisinin and artemisinincombination therapies had improved treatment options, while work on a Gatessponsored malaria vaccine was progressing apace, with a phase III trial due to start shortly. Moreover, 'eradication' was now defined loosely, with a degree of ambiguity that allowed diverse stakeholders to attach their own interpretations to the concept. It could refer to the vision of a world free of the malaria parasite the "permanent reduction to zero of the worldwide incidence of infection caused by human malaria parasites," in the WHO definition - but it could also be taken to mean cessation of disease transmission in individual countries or large geographical regions - what would become known as the strategy of "shrinking the malaria map" by eliminating the parasite "from the endemic margins inwards" (Feachem et al 2010), a still difficult but achievable objective. Margaret Chan, who as Director of the WHO had immediately endorsed the Gates Foundation's policy change, described the goal as "elimination-slash-eradication, depending on the availability of tools" (quoted in Roberts and Enserink 2007).

This implied relationship between the goal of eradication and the availability of tools made the new Gates vision very appealing across the board. The global eradication campaign of the 1950s and 1960s had been driven by the 
conviction that the tools necessary for eradication - DDT for the elimination of the mosquito vector and chloroquine for the elimination of blood-stage parasites in human hosts - were already at hand. Eradication was thought to be a matter of deploying these existing methods with ruthless efficiency and speed, before mosquitoes and parasites could develop resistance to these compounds (Packard 2007; Stepan 2013).

In contrast, the Gates Foundation's new focus on eradication came accompanied by the admission that the tools necessary to achieve this goal did not yet exist. The call for a new global eradication effort was in fact indistinguishable from the call to develop the technologies - insecticides, drugs, and vaccines - that would be required to make eradication possible. As Melinda Gates had noted in her speech, a more ambitious and more global objective would serve to energize the donor and scientific communities. "The goal of eradicating malaria has the power to create great expectations, grand efforts, and record funding" (Gates 2007). This is how the Foundation's change of strategy was interpreted: as a promise to do whatever it took to bring forth the new biomedical technologies that would make the goal of eradication feasible (Cueto 2013; Packard 2016). Considering that by 2007 the Gates Foundation was well established as the main funder of research on new malaria therapeutics, and was widely seen as the philanthropy with the deepest pockets, many in the scientific community who were skeptical of the feasibility of eradication, or of the wisdom of proclaiming it as the overarching goal of international efforts against malaria, welcomed the substantial institutional and financial commitment to research and development that the new agenda was thought to represent.viii

The change of focus had immediate implications for antimalarial drug discovery, and these were teased out by the Malaria Eradication Research Agenda initiative (malERA), a series of expert groups convened by the Gates Foundation to formulate new investment priorities. The malERA Consultative Group on Drugs noted the need "to revive the earlier paradigm and again think about malaria drugs and other interventions in terms of their impact on malaria infection and transmission in addition to their use in the prevention and treatment of malaria disease" (Alonso et al 2011). This shift in emphasis, from 
exclusively addressing the clinical manifestations of the disease to confronting the parasite's life-cycle in its entirety, implied a reorientation of R\&D efforts towards "new antimalarial drugs developed specifically for elimination indications" (ibid). The Consultative Group underscored the urgency of two such indications: drugs able to kill the sexual form of the parasites (gametocytes) in order to block transmission from human to mosquito, and drugs effective against dormant $P$. vivax parasites in the liver. If the goal was to stop transmission, and not simply to treat the symptoms of disease, these new drugs would need to be safe enough to be administered to asymptomatic individuals, probably in the context of mass drug administration campaigns.

Measured against these new goals, tafenoquine appeared in a promising light - not as a potential new prophylactic against falciparum malaria, however, but in its original guise as a curative treatment for vivax. While vivax malaria has historically been considered a 'benign' form of the disease, at least in comparison to falciparum malaria, new evidence was beginning to show the hidden burden, in terms of morbidity and mortality, of P. vivax infection (Price et al 2007). Furthermore, from an elimination standpoint vivax malaria poses an exceedingly demanding challenge. The parasite is adapted to a greater range of temperatures and has a wider geographical distribution than P. falciparum, its sexual forms develops (and can be transmitted from human to mosquito) before any clinical symptoms of infection appear, and it requires less time to complete its life cycle within the mosquito, which reduces the effectiveness of vector control measures. Last but not least, unlike P. falciparum, P. vivax presents a latent liver-stage form that can trigger blood-stage infection - and restore infectivity - long after the initial inoculation. The hypnozoite, long an obscure and poorly understood biological form, emerged now as an object of unprecedented pharmacological significance: it was "part of the front-line" in the battle against malaria, the "hidden obstacle" that had to be tackled for long-lasting elimination to stand a chance (Wells et al 2010)

Tafenoquine was the only molecule in development with proven efficacy against hypnozoites, and given the parlous state and secular neglect of research on P. vivax (there was, for instance, no continuous culture system for the parasite, and no in vitro system with which to assess the efficacy of new 
molecules on liver-stage parasites), it was very unlikely that an alternative drug with a similar level of hypnozoitocidal activity would come along anytime soon. Tafenoquine's long half-life meant that it might be possible to achieve radical cure with a single dose of the drug, which would greatly facilitate its eventual deployment in mass drug administration campaigns. Finally, the fact that the molecule had been in development for decades also worked to its advantage, for it meant that it had already been tested extensively in patients (about 3,000 of them, if we aggregate the populations of all the clinical studies conducted since the early 1990s), and that its clinical evaluation could be completed in a relatively short period of time.

In sum, a few years after it was declared a failed investigational drug, tafenoquine re-appeared as a promising 'new' drug - indeed as the ideal antimalarial. The molecule had not changed, but malaria had: it was now seen as a global challenge, with P. vivax at its center, and "elimination-slash-eradication" as the goal against which candidate drugs and pharmaceutical strategies were assessed.

\section{Tafenoquine and the new philanthropic-industrial complex}

A few months after the 2007 Gates Malaria Forum, MMV's External Scientific Advisory Committee (ESAC) agreed to include tafenoquine in the organization's portfolio of projects, and to collaborate with GSK in the development of the molecule as a new radical curative treatment for vivax malaria. The adoption of tafenoquine as a strategic project aligned MMV's drug discovery portfolio with the new priorities of the Gates Foundation, its largest funder. It also meant that MMV would potentially be in a position to register a new drug in a relatively short period of time. As a former member of ESAC puts it: "MMV was now at the point where they could take on a project like this. And it would round out their portfolio better than what they had going on at the time. And it was also latestage development, which is always very attractive: that you may be able to invest a few years and actually have a product that comes out of it pretty quickly." 
The co-development agreement between GSK and MMV differed significantly from the partnership that SmithKline Beecham and WRAIR had established in the 1980s. For one, most of the funding would now be coming from a third party, the Gates Foundation, via the Foundation's grants to MMV. In exchange, GSK agreed to develop tafenoquine on a "not-for profit, not-for-loss" basis, and expressed a commitment to making it available at affordable prices in disease-endemic countries. The U.S. Army would no longer be directly involved in the design or evaluation of the clinical program, but in recognition of the interest and past investments of the American military GSK agreed to seek registration of the drug with the FDA in the first instance, so that it would be available for use by U.S. military personnel as soon as possible. ${ }^{\text {ix }}$

The most significant issue looming over the development of tafenoquine was its toxicity in individuals with low levels of G6PD activity. When the molecule was being positioned as a new prophylactic for Western travelers this question could be bypassed, since it was assumed that most users would have access to professional G6PD diagnosis in their home countries. Now, however, the drug was being developed for the therapeutic benefit of individuals living in malaria-endemic countries. G6PD deficiency is especially common in some of these areas - it is thought to affect on average 8 per cent of the population in those regions, or about 400 million people, with significant variations in the severity of the deficiency depending on the genotypic variant (Howes et al 2012). Furthermore, some forms of G6PD deficiency appear to be associated with protection against severe $P$. falciparum infection. In other words, not only could tafenoquine be toxic to hundreds of millions of potential users, but it might be most dangerous to those with the best natural defenses against the disease. These considerations brought into sharper relief the significant gaps that had long existed in the scientific understanding of G6PD deficiency, and dramatically raised the bar in terms of identifying a safe dosing regimen for the drug (Mueller et al 2009). ${ }^{x}$

The first clinical trial co-sponsored by GSK and MMV was a phase I doseescalating study, launched in Thailand in 2009, intended to assess the levels of hemolysis caused by different quantities of tafenoquine in adults with moderately low levels of the G6PD enzyme. The study found that a 300mg dose 
posed no higher risk of hemolysis in this population than the standard primaquine treatment. It also concluded that, in order to limit the risk of hemolysis, administration of the drug in doses higher than $100 \mathrm{mg}$ should be preceded by a diagnosis of the G6PD status of the individual. The next phase of the clinical evaluation focused on the ability of the molecule to prevent relapse. In 2011, GSK and MMV launched a double-blind, randomized phase IIb trial to compare the efficacy of four alternative tafenoquine regimens (a single dose of $50 \mathrm{mg}, 100 \mathrm{mg}, 300 \mathrm{mg}$ or $600 \mathrm{mg}$ ) with a 2-week course of primaquine. Conducted simultaneously in Peru, Brazil, Thailand and India, the trial excluded individuals with low or moderately low levels of GSPD activity, pregnant and lactating women, and in general any women thought to be of "child-bearing potential."

The conclusion of this study marked a pivotal moment in the development of the molecule. 89.2 per cent of the patients who had received a single 300mg dose of tafenoquine were relapse-free six months after administration of the drug (the percentage increased only to 91.9 per cent with a $600 \mathrm{mg}$ dose), compared with 77.3 per cent of the patients who had received a 2 week course of primaquine. On the basis of this evidence, tafenoquine was granted 'orphan drug' status by the FDA in January 2013, and received 'breakthrough therapy' designation from the same regulator a few months later, thereby guaranteeing an accelerated review process. Discussing the results of these recent trials in The Lancet, Ric Price and François Nosten summarized the cautiously hopeful prospects of the molecule at this time. 'If subsequent clinical trials can confirm safety and comparative efficacy against current treatment options, and the drug can be deployed widely to patients, then a single-dose tafenoquine radical cure has potential to transform $P$. vivax therapeutics and become a major contributor to malaria elimination' (Price and Nosten 2014: 1021).

As the evaluation of the molecule progressed apace, tafenoquine's transformative potential came to depend on whether individuals at risk of hemolytic toxicity could be quickly and accurately screened prior to administration. "The great promise of tafenoquine," wrote vivax expert Kevin Baird, "emphasizes the urgency of solving the problem of G6PD diagnosis that 
would otherwise deny most patients access to the therapy" (in Tanner et al 2015). Several G6PD diagnostic platforms were available, but they all required laboratory conditions, complex equipment, and extensive technical skills. The safe use of tafenoquine in endemic regions, however, particularly as an elimination tool, would bring the drug to areas with no access to professional medical staff or laboratory facilities. It was therefore imperative to develop fieldadapted, point-of-care diagnostic tools, capable of operating in the absence of any supporting analytical infrastructure. These, moreover, would need to be highly accurate: unlike primaquine, tafenoquine would be administered in a single dose, making it impossible to discontinue treatment once symptoms of hemolysis appeared. Finally, tafenoquine would be distributed as a GSK-branded product, and the company was exceedingly risk-averse when it came to the possibility of being responsible for serious adverse events associated with a drug it considered primarily an asset in its corporate social responsibility strategy. As a result of all these considerations, GSK and MMV agreed that administration of tafenoquine should be guided by a quantitative point-of-care diagnostic device able to accurately identify individuals with less than 70 per cent of the normal level of G6PD activity. Anyone under that threshold would in principle be denied access to the drug.

The approach taken to the development of these diagnostic devices has replicated the pattern of philanthropic-industrial collaboration adopted for the evaluation of the molecule. The Gates Foundation once again stepped in and, together with the UK Department for International Development (DFID), funded most of the development work, this time through the Seattle-based non-profit Program for Appropriate Technology in Health (PATH). PATH defined a set of criteria for future point-of-care diagnostic tools, including their affordability in malaria-endemic countries, and established the technical infrastructures (such as a global repository of cryopreserved blood samples characterized for different levels of G6PD activity) to assist commercial firms in the development of proprietary testing platforms.

The evaluation of tafenoquine reached its final milestone with the launch in 2014 of a multi-centre, randomized, double-bind phase III clinical trial. The first part of the study compared the efficacy of a single $300 \mathrm{mg}$ dose of 
tafenoquine with a 14-day course of primaquine in individuals with confirmed vivax infection. The second part of the study assessed reduction of hemoglobin levels (an indicator of hemolysis) after administration of $300 \mathrm{mg}$ of tafenoquine to vivax-infected patients. Headline results, announced in the summer of 2017, indicated that the single-dose of tafenoquine had largely the same radical curative efficacy as the two-week course of primaquine, while exposing patients to no higher risk of hemolysis. On the basis of these results, in November 2017 GSK filed a New Drug Application with the FDA seeking approval for the use of a single $300 \mathrm{mg}$ dose of tafenoquine for the radical cure of vivax malaria in patients 16 years of age and older. In July 2018, the FDA approved the application, and authorized GSK to commercialize the new drug under the trade name Krintafel. ${ }^{\mathrm{xi}}$

\section{Conclusion: conjugating a new global malaria}

Forty years after it was first identified as a promising molecule, tafenoquine is now ready to be used for the treatment of vivax malaria. Despite the limitations it shares with other 8-aminoquinoline compounds - its mechanism of action remains unknown, it has not been tested in children, and it is contraindicated in pregnant women - the drug is eagerly awaited by many in the malaria control community who see in it a valuable option for the management of vivax infections and a potent new weapon in future malaria eradication campaigns. Sue Desmond-Hellmann, CEO of the Gates Foundation, welcomed the FDA's approval of Krintafel as "a major milestone along the road to ending the scourge of malaria for good." Hinting at the imminent deployment of the drug in Brazil, she suggested the start of a continent-wide eradication effort. "The next domino to fall on the path to eradication can be the Americas. This is an opportunity we must seize."xii

Looking back on these four decades of development, it is easy to lament the missed opportunities and multiple delays that kept the molecule for so long in the limbo of not-quite-yet-drugs. Alan Magill, former Director for Malaria at the Gates Foundation and a researcher who was instrumental to the successful development of the molecule (he was director of drug development at WRAIR 
and provided a vital link between the U.S. Army's antimalarial drug discovery program and the contemporary Gates-sponsored push for eradication) used to claim that tafenoquine ought to be a Harvard Business School case study in how not to develop a new drug. xiii The therapeutic potential of the molecule had been apparent from the start and was clinically demonstrated in the 1990s. The challenge of hemolytic toxicity in G6PD-deficient individuals was well known, if not well understood, and could have been addressed much earlier in the development process. The long delay in the fulfillment of the molecule's promise, he argued, had nothing to do with its pharmacokinetic qualities. It was due to changes in the political and economic environment that undermined institutional support for the drug within the organisations responsible for its development.

The ebbing fortunes of tafenoquine help us identify three critical junctures in the political economy of malaria drug discovery since the 1970s. One is the transition from the once all-powerful military drug discovery program of the US Army towards a new regime that relied primarily on the commercial calculations of large pharmaceutical companies. This transition, evident after the end of the Vietnam War, involved a narrowing of pharmacological focus on a single indication, prophylaxis against falciparum malaria, and resulted in drug development programmes geared towards the requirements and resources of Western visitors to disease-endemic countries.

The second point of transition is the rise, around the turn of the century, of a new 'global health' vision for malaria therapeutics. Pharmaceutical innovation was re-energized and re-oriented towards the urgent clinical needs of those living in malarious regions, and was infused with a humanitarian ethos that set itself in explicit opposition to the commercial and military rationales that had dominated malaria drug discovery during most of the twentieth century. Prophylaxis became 'a dirty word,' and tafenoquine was tainted by its association with a regime of pharmaceutical $R \& D$ that was seen to have failed chronically vulnerable populations.

The revival of tafenoquine after 2007 marks a third point of transition, this time between two distinct phases within the global health era: the initial period of chemotherapeutic humanitarianism, reactive in origin and palliative in aspiration, and a latter period, still underway, where research efforts are guided 
by the goal of global eradication, an essentially prospective and promissory horizon. Each of these two moments in the global health regime has advanced a different pharmaceutical vision of malaria: the humanitarian phase focused on $P$. falciparum, specifically on the suppression of the asexual blood-stage parasites that cause clinical attacks, while the shift towards eradication has re-directed attention towards P. vivax and emphasized chemical agents with "eliminationspecific" indications. ${ }^{x i v}$

Tafenoquine is the first 'new' drug to fully materialize this latter vision of malaria. In the form of the recently approved Krintafel, it conjugates the most distinctively global of the malarias brought forth in the global health era. It attacks $P$. vivax, the most geographically widespread of the human malaria parasites, and reverses the consideration of vivax as a "benign" form of the disease, thus greatly enlarging the target population for pharmaceutical interventions. It conjugates a malaria, moreover, that is defined by levels of parasite prevalence in symptomatic and asymptomatic individuals, as much as by the morbidity and mortality the parasite causes in patients. A malaria, in other words, for which treatment increasingly means cure, and for which cure is radicalized and taken to mean the complete elimination of parasites from the body. Individual treatment becomes a means towards a collective end: to stop disease transmission by wiping out the "human reservoir" of latent liver-stage parasites. This form of collective pharmaceutical care is seen, in turn, as the best, indeed the only way of enduringly protecting individuals from infection and disease. This is, in other words, a cartographic malaria, for which success is measured by the ability to "shrink the malaria map" and reduce the territorial footprint of the parasite.

The global, inclusive nature of the malaria tafenoquine conjugates is qualified, however, by trends that fragment the collective the new drug is expected to serve. The molecule's toxicity excludes from its use pregnant women and young infants, the two groups most vulnerable to severe malaria and the key beneficiaries of chemotherapeutic humanitarianism. ${ }^{\mathrm{xv}}$ The decision to base administration of the drug on prior diagnosis of the individual's G6PD status will further fragment the target population along genotypic and phenotypic lines, and the choice of a high (70 per cent) threshold of G6PD activity to identify those at 
risk of hemolysis will potentially deny access to the drug to a significant proportion of the population in malaria-endemic regions, specifically women with 'mild' or 'intermediate' levels of enzyme deficiency.

These are some of the aggregating and disaggregating forces tafenoquine will bring forth, chemo-political traits of the drug that exemplify the "possibilities and inequalities" that this new pharmaceutical conjugation of malaria engenders (Biehl 2007). The standout achievement of a decade of malaria drug discovery dominated by a philanthropic-industrial vision, the drug also anticipates the obstacles that will compromise and perhaps even undermine the promise it embodies. By instantiating a pharmaceutical strategy towards eradication, Krintafel sets in motion a set of formidable counterforces: the emergence of strains of $P$. vivax resistant to the chemical; the assortment of "social" and "cultural" factors expected to hinder mass administration of the drug, especially in the hard-to-reach communities that often represent the last barrier to regional elimination; and, last but not least, that peculiar ailment of the global health regime, an ailment that is intrinsic to its distinctive political economy: "donor fatigue," the feared lapse in the commitment of the handful of Western actors whose financial support sustains this promissory technological enterprise.

\section{Acknowledgments}

I would like to thank Kevin Baird, Rosalind Howes, Ann Kelly and Claas

Kirchhelle for comments on an earlier version of this article.

\section{Funding:}

This research was supported by the European Research Council under the European Community's Seventh Framework Programme (FP7/2007-2013)/ERC grant agreement no. 263447 (BioProperty).

\section{Biographical note:}

Javier Lezaun in Associate Professor in the School of Anthropology and Museum Ethnography and Director of the Institute for Science, Innovation and Society at the University of Oxford. 


\section{References}

ALONSO, P. L., BASSAT, Q., BINKA, F. et al (2011). A research agenda for malaria eradication: drugs. PLoS medicine, 8(1).

BAIRD, J. K. (2007). Neglect of Plasmodium vivax malaria. Trends in parasitology, 23(11), 533-539.

BAIRD, J. K. (2017). Malaria control by commodities without practical malariology. BMC public health, 17(1), 590.

BIEHL, J. (2007). Pharmaceuticalization: AIDS treatment and global health politics. Anthropological Quarterly, 1083-1126.

COLEMAN, R. E. (1990). Sporontocidal activity of the antimalarial WR-238605 against Plasmodium berghei ANKA in Anopheles stephensi. The American Journal of Tropical Medicine and Hygiene, 42(3), 196-205.

CROFT, A. M. (2007). A lesson learnt: the rise and fall of Lariam and Halfan. Journal of the Royal Society of Medicine, 100(4), 170-174.

CUETO, M. (2013). A return to the magic bullet?: malaria and global health in the twenty first century. In J. BIEHL and A. PETRYNA (eds.) When People Come First: Critical Studies in Global Health. Princeton: Princeton University, 30-53.

DOW, G. S., MAGILL, A. J., \& OHRT, C. (2008). Clinical development of new prophylactic antimalarial drugs after the 5th Amendment to the Declaration of Helsinki. Therapeutics and clinical risk management, 4(4), 803.

ECKS, S. (2008). Global pharmaceutical markets and corporate citizenship: The case of Novartis' anti-cancer drug Glivec. BioSocieties, 3(2), 165-181.

ECKS, S., \& HARPER, I. (2013). Public-private mixes: the market for antituberculosis drugs in India. In J. BIEHL and A. PETRYNA (eds.) When People Come First: Critical Studies in Global Health. Princeton: Princeton University, 3053. When people come first: critical studies in global health, 252-275.

FEACHEM, R. G., PHILLIPS, A. A., HWANG, J. et al (2010). Shrinking the malaria map: progress and prospects. The Lancet, 376(9752), 1566-1578.

GALAMBOS, L. (2001). "Global Oligopoly, Regional Authority, and National Power: Crosscurrents in Pharmaceuticals Today and Tomorrow." In H. Kettler (ed.) Consolidation and Competition in the Pharmaceutical Industry. London: Office of Health Economics. 
GATES, Melinda French (2007). Malaria Forum Keynote Address. 17 October 2017. Available at https://www.gatesfoundation.org/mediacenter/speeches/2007/10/melinda-french-gates-malaria-forum

HALE, B. R., OWUSU-AGYEI, S., FRYAUFF, D. J. et al (2003). A randomized, doubleblind, placebo-controlled, dose-ranging trial of tafenoquine for weekly prophylaxis against Plasmodium falciparum. Clinical Infectious Diseases, 36(5), 541-549.

HECHT, G. (2002). Rupture-talk in the nuclear age: conjugating colonial power in Africa. Social Studies of Science, 32(5-6), 691-727.

HOWES, R. E., PIEL, F. B., PATIL, A. P. et al (2012). G6PD deficiency prevalence and estimates of affected populations in malaria endemic countries: a geostatistical model-based map. PLoS medicine, 9(11), e1001339.

LAKOFF, A. (2010). Two regimes of global health. Humanity: An International Journal of Human Rights, Humanitarianism, and Development, 1(1), 59-79.

LESS, B., FAUCHER, J. F., MISSINOU, M. A. (2000). Malaria chemoprophylaxis with tafenoquine: a randomised study. The Lancet, 355(9220), 2041-2045.

LEZAUN, J., \& MONTGOMERY, C. M. (2015). The pharmaceutical commons: Sharing and exclusion in global health drug development. Science, Technology, \& Human Values, 40(1), 3-29.

MCGOEY, L., REISS, J., \& WHALBERG, A. (2011). The global health complex. BioSocieties, 6(1), 1-9.

MILHOUS, W. K. (2001). Development of new drugs for chemoprophylaxis of malaria. Médecine tropicale, 61(1), 48-50.

MORAN, M., GUZMAN, J., ROPARS, A. L. et al (2009). Neglected disease research and development: how much are we really spending? PLoS medicine, 6(2), e1000030.

MUELLER, I., GALINSKI, M. R., BAIRD, J. K. et al (2009). Key gaps in the knowledge of Plasmodium vivax, a neglected human malaria parasite. The Lancet infectious diseases, 9(9), 555-566.

PACKARD, R. M. (2007). The making of a tropical disease: a short history of malaria. Baltimore: Johns Hopkins University Press.

PACKARD, R. M. (2016). A History of Global Health: Interventions into the Lives of Other People. Baltimore: Johns Hopkins University Press.

PETERS, W., ROBINSON, B. L., \& MILHOUS, W. K. (1993). The chemotherapy of rodent malaria. LI. Studies on a new 8-aminoquinoline, WR 238,605. Annals of Tropical Medicine \& Parasitology, 87(6), 547-552. 
PETERS, W. (1999). The evolution of tafenoquine-antimalarial for a new millennium? Journal of the Royal Society of Medicine, 92(7), 345-352.

PRICE, R. N., TJITRA, E., GUERRA, C. A. et al (2007). Vivax malaria: neglected and not benign. The American journal of tropical medicine and hygiene, 77(6_Suppl), 79-87.

PRICE, R. N., \& NOSTEN, F. (2014). Single-dose radical cure of Plasmodium vivax: a step closer. Lancet (London, England), 383(9922), 1020.

ROBERTS, L., \& ENSERINK, M. (2007). Did they really say... eradication? Science, 318(5856), 1544-1545.

SAMSKY, A. (2012). Scientific sovereignty: how international drug donation programs reshape health, disease, and the state. Cultural Anthropology, 27(2), 310-332.

SHANKS, G. D., OLOO, A. J., ALEMAN, G. M. et al (2001). A new primaquine analogue, tafenoquine (WR 238605), for prophylaxis against Plasmodium falciparum malaria. Clinical infectious diseases, 33(12), 1968-1974.

SLATER, L. B. (2009). War and disease: biomedical research on malaria in the twentieth century. New Brunswick: Rutgers University Press.

SMOAK, B. L., DEFRAITES, R. F., MAGILL, A. J. et al (1997). Plasmodium vivax infections in US Army troops: failure of primaquine to prevent relapse in studies from Somalia. The American Journal of Tropical Medicine and Hygiene, 56(2), 231234.

STEPAN, N. L. (2013). Eradication: Ridding the World of Diseases Forever? London: Reaktion Books.

TANNER, M., GREENWOOD, B., WHITTY, C. J. et al (2015). Malaria eradication and elimination: views on how to translate a vision into reality. BMC medicine, 13(1), 167.

TROULLIER, P., \& OLLIARO, P. L. (1998). Drug development output from 1975 to 1996: what proportion for tropical diseases? International Journal of Infectious Diseases, 3(2), 61.

U.S. ARMY (1992) Review of DoD Malaria Research Programs. Army Medical Research and Development Command. Fort Detrick: Frederick, Maryland

WELLS, T. N., BURROWS, J. N., \& BAIRD, J. K. (2010). Targeting the hypnozoite reservoir of Plasmodium vivax: the hidden obstacle to malaria elimination. Trends in parasitology, 26(3), 145-151.

WHITE, N. J., NOSTEN, F., LOOAREESUWAN, S. et al (1999). Averting a malaria disaster. The Lancet, 353(9168), 1965-1967. 
WHO (1999). World Health Report, 1999. Geneva: World Health Organisation.

WINZELER, E. A. (2008). Malaria research in the post-genomic era. Nature, 455(7214), 751-756.

\footnotetext{
i Between 1975 and 1998, and leaving aside artemisinin and its derivatives (which emerged out of research conducted at the Chinese Academy of Military Medical Sciences), only three new antimalarials were licensed: mefloquine, halofantrine, and a fixed combination of atovaquone and proguanil (Trouiller and Olliaro 1998). Two of those, mefloquine and halofantrine, were of limited value due to their toxic effects, while the fixed combination of atovaquone and proguanil, marketed under the trade name Malarone, was too expensive to be available for general use in malaria-endemic regions.

ii The potential of primaquine to provoke acute hemolysis was known at the time of its development, since this toxicity issue is common to other molecules in the 8-aminoquinoline class, such as pamaquine or pentaquine, which had been widely tested in the 1940s. The link between hemolytic risk and G6PD deficiency was established in 1956.

iii Under U.S. law, the American military is only allowed to use pharmaceutical products approved by the FDA and in strict accordance with the label.

iv A Randomized, Double Blind, Placebo Controlled Evaluation of Weekly Tafenoquine (WR 238605/SB252263) Compared to Mefloquine for Chemosuppression of Plasmodium Falciparum in Western Kenya. ClinicalTrials.gov Identifier: NCT02488980.

${ }^{v}$ By the standards of the sector, the new company had an unusually large portfolio of antimalarials At the time of their merger Glaxo Wellcome and SmithKline Beecham commercialized chloroquine, chlorproguanil, dapsone, sitamaquine, halofantrine, and atovaquone-proguanil, and had several other molecules and combinations in development. In its review of the proposed merger, the U.S. Federal Trade Commission raised the combined portfolio of antimalarials as a possible antitrust concern.

vi It was not incidental that the two previous antimalarials to emerge from the U.S. Army's drug discovery program, mefloquine (Larian) and halofantrine (Halfan), had been found to cause serious adverse events soon after their licensure, and that some thought these two drugs had been authorized "on the basis of an incomplete knowledge base, and at too early a stage in the normal cycle of drug development" (Croft 2007: 172).

vii Although in the past the WHO had endorsed the use of weekly prophylaxis to protect children and pregnant women in endemic areas, by the late 1990s this option had lost support due to concerns about its affordability, the impact on the development of drug-resistant parasites, and the impairment of natural immunity. The sensitivity triggered by the term "prophylaxis" is evident in the coining, around this time, of the concept of "intermittent preventive treatment" to describe pharmaceutical interventions that blurred the distinction between prophylactic effect (prevention of blood-stage infection) and treatment effect (elimination of parasites circulating in the bloodstream).

viii In 2008, for instance, the Foundation would spend US $\$ 124$ million on malaria-related R\&D activities, out of the total of $\$ 375$ million that public and philanthropic funders provided that year (Moran et al 2009). This figure underestimates the influence of the Foundation, however, since the second and third largest sponsors, the U.S. National Institutes of Health and the U.S. Department of Defense, were much more constrained in the sort of projects they were able to support. The Foundation's donations were crucial in the area of drug development - in 2008 it was the source of 60 per cent of MMV's funding, for instance.

ix A parallel submission was made to the Australian Therapeutic Goods Administration. The U.S. Army retained the rights to the development of the molecule for vivax and falciparum prophylaxis. These rights were licensed to the firm $60^{\circ}$ Pharmaceuticals.

$x$ GSK had recently experienced the failure of the antimalarial Lapdap (a new combination of chlorproguanil and dapsone), due partly to adverse side effects in G6PD-deficient patients. In 2008, as the development of tafenoquine was getting underway, GSK and MMV halted the joint
} 
development of Dacart, a combination of chlorproguanil, dapsone and artesunate, after a phase III trial showed a significant reduction of hemoglobin in patients with low G6PD levels.

xi The FDA granted GSK a tropical disease priority review voucher, which the company can use to accelerate the review of any other drug candidate. A few days later the FDA approved an application from $60^{\circ}$ Pharmaceuticals to market, under the trade name Arakoda, $100 \mathrm{mg}$ tablets of tafenoquine for malaria prophylaxis.

xii https://twitter.com/SueDHellmann/status/1020428603408855040 [20 July 2018]

xiii Personal communication, summer of 2014.

xiv These two distinct periods within the global health era correspond roughly to the two regimes of global health identified by Andrew Lakoff (2010): a humanitarian biomedicine oriented towards the protection of individual lives immediately threatened by existing disease, and a vision of global health security driven by the desire to prevent future outbreaks. The case of malaria suggests that the regime of global health security extends beyond the anticipation and prevention of emerging (or yet to emerge) diseases and encompasses also efforts to eliminate endemic pathogens from large areas of the world. The example of tafenoquine proves that the same pathogen can fall under either regime, and switch from one to another in a relatively short period of time, especially when a handful of global actors dominate the funding landscape and can quickly change research and intervention priorities.

${ }^{\mathrm{xv}}$ An open-label study of the safety and efficacy of lower doses of tafenoquine in pediatric subjects ( 6 months to 16 years of age) was launched in Colombia, Thailand and Vietnam in 2017. 